\title{
MicroRNA-483-5p Modulates the Expression of Cartilage-Related Genes in Human Chondrocytes through Down-Regulating TGF- $\beta 1$ Expression
}

\author{
Ronghua Xu, ${ }^{1,2}$ Jiayi Li, ${ }^{1}$ Bo Wei, ${ }^{1}$ Weiling Huo ${ }^{2}$ and Liming Wang ${ }^{1}$ \\ ${ }^{1}$ Department of Orthopaedic Surgery, Nanjing First Hospital, Nanjing Medical University, Nanjing, Jiangsu, China \\ ${ }^{2}$ Department of Orthopaedic Surgery, Xuzhou Central Hospital, Xuzhou, Jiangsu, China
}

Transforming growth factor- $\beta 1$ (TGF- $\beta 1$ ) has been reported to improve chondrocytes phenotype and function. The expression levels of microRNA-483-5p (miR-483-5p), a potential regulator of TGF- $\beta$ signaling pathway, were elevated in chondrocytes of patients with osteoarthritis. In this study, we aimed to explore the role of miR-483-5p for the expression of cartilage-related genes in chondrocytes. Human chondrocytes were harvested from femoral condyle and tibial plateau of different donors $(n=10)$ following amputation due to sarcomas not involving the joint space. The expression levels of miR-483-5p and TGF- $\beta 1$ mRNA were measured by qRT-PCR. Runt-related transcription factor 2 (RUNX2) is a transcription factor involved in chondrocyte differentiation, and type II collagen-degrading matrix metalloproteinase13 (MMP13) contributes to cartilage degradation. qRT-PCR was also used to measure the levels of RUNX2 and MMP-13 mRNAs, as well as type II collagen alpha 1 (COL2A1) and aggrecan mRNAs. COL2A1 and aggrecan are major cartilage extracellular matrix proteins that are essential for normal cartilage function. The expression levels of miR-483-5p were significantly increased in chondrocytes from Passage 0 to 2 , whereas the expression levels of TGF- $\beta 1$ mRNA were marginally decreased. Passage 1 chondrocytes were employed for following experiments. MiR-483-5p overexpression reduced TGF- $\beta 1$ expression, while miR-483-5p knockdown dramatically elevated TGF- $\beta 1$ expression both at mRNA and protein levels. Further, miR-483-5p overexpression significantly decreased the levels of COL2A1 and aggrecan mRNAs, and increased those of RUNX2 and MMP13 mRNAs, by down-regulating TGF- $\beta 1$ expression. These findings suggest that modulating miR-483-5p expression may contribute to maintaining the cartilage tissues.

Keywords: cartilage repair; chondrocyte; miR-483-5p; osteoarthritis; TGF- $\beta 1$.

Tohoku J. Exp. Med., 2017 September, 243 (1), 41-48. C 2017 Tohoku University Medical Press

\section{Introduction}

Articular cartilage is a flexible connective tissue covering the surfaces of the synovial joints (Ashraf et al. 2016) and enables weight bearing and near friction-free movement in the joints of the human body (Martinez-Sanchez et al. 2012). Cartilage is a unique avascular, aneural, and alymphatic tissue that lacks resident progenitor stem cells, and thus has little self-healing potential once injured (Goessler et al. 2005; Candela et al. 2014). Chondrocytes, the primary component of articular cartilage, are essential for the production of extracellular matrix (ECM) and maintenance of cartilage structure and function (Caron et al. 2012). In addition, chondrocytes are recently demonstrated to be important in preventing articular degeneration (Tang et al. 2017).

Transforming growth factor- $\beta$ (TGF- $\beta$ ) plays a crucial role in the induction of chondrogenesis and the maintenance of chondrogenic differentiation. Among TGF- $\beta$ superfamily, the most widely studied isoform in chondrocytes was transforming growth factor- $\beta 1$ (TGF- $\beta 1$ ), which has been clearly shown to be implicated in chondrogenic differentiation (Worster et al. 2000). TGF- $\beta 1$ is implicated in a complicated signaling mechanism that ultimately contributes to the maintenance of the chondrocytic characteristics during in vitro culture, through promoting cell proliferation and ECM protein synthesis as well as protecting normal morphology (Blaney Davidson et al. 2007; Qureshi et al. 2007). TGF- $\beta 1$ was also reported to promote the synthesis of sulfated glycosaminoglycans and collagen in chondrocytes, suggesting its potential role for articular cartilage repair (Davies et al. 2008). However, little is known about the mechanisms of controlling expression of TGF- $\beta 1$ itself.

MicroRNAs (miRs) are small non-coding RNAs with

Received January 17, 2017; revised and accepted August 17, 2017. Published online September 16, 2017; doi: 10.1620/tjem.243.41. Correspondence: Liming Wang, Ph.D., Department of Orthopaedic Surgery, Nanjing First Hospital, Nanjing Medical University, No. 68 Changle Road, Nanjing, Jiangsu 210006, China.

e-mail:wang_lm@sohu.com 
21-23 nt in length that regulate expression of target genes and play crucial roles in various cellular processes (Bartel 2004; Martinez-Sanchez et al. 2012). Recent studies have shown the importance of miRNAs in chondrocyte function (Díaz-Prado et al. 2012). MiR-145 expression increased with loss of the differentiated chondrocyte phenotype and regulated levels of key cartilage genes in human chondrocytes (Martinez-Sanchez et al. 2012). miR-140 was elevated and acted as a negative feedback regulator of type II collagen-degrading matrix metalloproteinase13 (MMP13) in interleukin-1 $\beta$ (IL-1 $\beta$ )-stimulated human articular chondrocyte C28/I2 cells (Liang et al. 2012). Overexpressing miR-483-5p suppressed several members of the mitogen activated protein kinase (MAPK) pathway, largely explaining the increased matrix production in bovine or human chondrocytes (Yang et al. 2015). MiR-483-5p was associated with several diseases such as adrenocortical carcinoma (Patterson et al. 2011), ovarian serous carcinoma (Yu et al. 2013), and multiple osteochondroma (Zuntini et al. 2010). Actually, miR-483-5p was also up-regulated in osteoarthritis chondrocytes and predicted to be associated with TGF- $\beta$ signaling pathway (Díaz-Prado et al. 2012). However, the role of miR-483-5p in chondrocytes remains unclear. Considering that TGF- $\beta 1$ has been implicated as an important mediator in promoting tissue repair through increased major articular cartilage matrix components (Zhu et al. 2015), we wondered whether miR-483-5p is involved in the regulation of TGF- $\beta 1$ expression in articular cartilage chondrocytes. Accordingly, here, we investigated the role of miR-483-5p and the relationship between miR-483-5p and TGF- $\beta 1$ in human cartilage chondrocytes.

\section{Materials and Methods}

\section{Isolation and culture of chondrocytes}

Human healthy cartilage samples were obtained from ten patients ( 6 males and 4 females with a mean age of 40 years and a range from 17 to 60 years, for full details, see Table 1) in Nanjing First Hospital. Written informed consent was obtained from all patients. This study was approved by the Ethic Committee of Nanjing First Hospital, Nanjing Medical University. All specimens were handled following the ethical and legal standards.

Cartilage was harvested from the femoral condyle and tibial plateau following amputation due to sarcomas not involving the joint space. In brief, non-diseased cartilage specimens were collected from the 10 different donors on the day of surgery under sterile conditions. After that, the specimens were rinsed with PBS and DMEM (Gibco, USA) containing penicillin $(100 \mathrm{U} / \mathrm{mL})$ and streptomycin $(100 \mu \mathrm{g} /$ $\mathrm{mL}$ ) (Gibco, USA). Cartilage samples were cut into $1-2 \mathrm{~mm}^{3}$ and incubated in $0.2 \%$ type II collagenase at $37^{\circ} \mathrm{C}$ with $5 \% \mathrm{CO}_{2}$. After 18 $\mathrm{h}$ of shaking, isolated chondrocytes were then filtered through a cell strainer, pelleted, and washed with DMEM complete medium. After that, cells were seeded at $1 \times 10^{4} / \mathrm{mL}$ in DEME with $10 \% \mathrm{FBS}$, and incubated at $37^{\circ} \mathrm{C}$ with $5 \% \mathrm{CO}_{2}$. These primary articular chondrocytes isolated from 10 different donors were labeled as Passage 0 (P0) cells. After serial subcultivation for 5-7 days, P0 cells were used as directly P1 cells and passaged for once more as P2 cells when they reached $80 \%$ confluency. Medium was changed every 3 days. In
Table 1. Donor details.

\begin{tabular}{ccc}
\hline Donor & Age & Sex \\
\hline 1 & 54 & female \\
2 & 38 & male \\
3 & 60 & male \\
4 & 47 & female \\
5 & 24 & male \\
6 & 17 & male \\
7 & 30 & male \\
8 & 52 & female \\
9 & 45 & female \\
10 & 33 & male \\
\hline
\end{tabular}

Human articular chondrocytes were isolated from 10 different donors. Non-diseased cartilage was harvested from knee following amputations due to osteosarcomas not involving the joint space.

total, we made the ten different preparations of articular chondrocytes.

Identification of chondrocytes

After incubation for $72 \mathrm{~h}$ on glass coverslips, P2 chondrocytes were rinsed with PBS and fixed by $10 \%$ paraformaldehyde for 30 min. After that, slips were stained with $1 \%$ toluidine blue (TB) at room temperature for $30 \mathrm{~min}$. Then coverslips were quickly washed by ethanol, dried, placed on glass slides, and sealed. Slides were then analyzed using a microscope.

\section{Construction of plasmids}

To knockdown miR-483-5p, scramble siRNA and miR-483-5p siRNA (si-miR-483-5p) were designed. To overexpress TGF- $\beta 1$ or miR-483-5p, the TGF- $\beta 1$ cDNA fragment or pri-miR-483-5p was cloned into pcDNA3.1 vector (Invitrogen, USA), generating pcDNA3.1-TGF- $\beta 1$ or pcDNA3.1-pri-miR-483-5p. The primers for cDNAs were as follows: TGF- $\beta 1,5$-CTAGTCTAGAATGCCGCC CTCCGGGCTGC-3' (forward) and 5'-CTAGATACTTCAGCTGCA CTTGCAGGAGCG-3' (reverse); and miR-483-5p, 5'-CCAAGC TTGTGAAATGGGCTCACAGGAT-3' (forward) and 5'-GCTCTA GATGTCCCTGAGCTTGGACTCT-3' (reverse). P1 chondrocytes isolated from 10 different donors were then transfected or co-transfected with the indicated constructs using Lipofectamine 2000 (Invitrogen, USA) according to the manufacturer's instructions.

\section{siRNA transfection}

The siRNA against miR-483-5p and scrambled siRNA were synthesized by a service provider (GenePharma Co., Ltd, Guangzhou, China). P1 chondrocytes isolated from 10 different donors were transfected with each siRNA using a transfection reagent (Lipofectamine 2000, Invitrogen, USA) according to the protocol from the manufacturer. The efficiency of siRNA to knock-down miR483-5p was determined by quantitative real-time PCR. 
RNA extraction and quantitative real-time PCR ( $q R T-P C R)$

Total RNA was extracted from human articular chondrocytes using TRIzol reagent (Invitrogen, USA) following the manufacturer's instructions and was reverse transcribed into cDNAs using the High Capacity cDNA reverse transcription kit (Applied Biosystems, USA). The cDNA template was amplified through qRT-PCR using SYBR Green PCR master mix by the ABI7900 system (Applied Biosystem, USA). The relative expression of miR-483-5p, TGF- $\beta 1$, COL2A1, aggrecan, RUNX2, and MMP13 were calculated by the $2^{-44 \mathrm{Ct}}$ method and normalized to the internal control GAPDH. The specific primers were as follows (Davies et al. 2008; Martinez-Sanchez et al. 2012; Li et al. 2014): miR-483-5p, 5'-GGAAGACGGGAGAAGAGA AGGG-3' (forward) and 5'-ATTGCGTGTCGTGGAGTCG-3' (reverse); TGF $\beta 1,5^{\prime}$-GCCCTGGACACCAACTACTGCTTC AGCTCC-3' (forward) and 5'-GCTGCACTTGCAGGAGCGCAC GATCATGTT-3' (reverse); aggrecan, 5'-CGTGCGCCCATCAACA-3' (forward) and 5'-ACACCTCCCCCTCAAGTCTGT-3' (reverse); COL2A1, 5'-GGAAGAGTGGAGACTACTGGATTGAC-3' (forward) and 5'-TCCATGTTGCAGAAAACCTTCA-3' (reverse); MMP13, 5'-TTACCAGACTTCACGATGGCATT-3' (forward) and 5'-TCGCCATGCTCCTTAATTCC-3' (reverse); RUNX2, 5'-CAG GCAGGTGCTTCAGAACTG-3' (forward) and 5'-GCATTCGTG GGTTGGAGAA-3' (reverse); and GAPDH, 5'-ACAACTTTGGTA TCGTGGAAGG-3' (forward) and 5'-GCCATCACGCCACAGT TTC-3' (reverse).

\section{Western blot analysis}

Human articular chondrocytes were harvested and lysed in RIPA buffer (Thermo Scientific, USA). The protein concentrations were determined by BCA assay. Subsequently, equal proteins were resolved by $10 \%$ SDS-PAGE gels and then transferred to PVDF membranes (Millipore, USA). After being blocked with 5\% non-fat dried milk, the membranes were incubated with primary antibodies against TGF- $\beta 1$ (Cell Signaling Technology, USA) overnight at $4{ }^{\circ} \mathrm{C}$, followed by secondary antibodies horseradish peroxidase-conjugated goat anti-rabbit IgG. GAPDH was used as an internal control. The protein was detected with an enhanced chemiluminescence kit (ECL kit, Pierce Biotechnology, IL) and the band intensity was analyzed by Image-Pro Plus 6.0 software.

\section{Statistical analysis}

The software of SPSS 17.0 (SPSS Inc., USA) was used for statistical analysis. Each experiment was performed as least three times. Data were presented as mean \pm standard deviation (SD). Differences between two groups were evaluated by Student's t-test. $P<0.05$ was considered statistically significant.

\section{Results}

Expression of miR-483-5p and TGF- $\beta 1$ mRNA in articular chondrocytes

Cellular morphology of chondrocytes obtained from 10 different donors was examined under inverted microscope during in vitro monolayer culture. Representative P0 chondrocytes were small, spherical, and diffusely distributed just after seeding. After cultivation for $72 \mathrm{~h}, \mathrm{P} 0$ chondrocytes exhibited dramatic proliferation. P1 and P2 cells proliferated rapidly and were sphere or polygonal shape, with only small proportion being long and narrow. It is widely known that toluidine blue (TB) can be bound with proteoglycan secreted by chondrocytes through polymer coupling, thus identify isolated chondrocytes ( $\mathrm{Li}$ et al. 2010). Here, P2 chondrocytes stained by TB displayed blue particles in the cytoplasm and dark blue round or oval nuclei (Fig. 1A).

We then performed qRT-PCR to detect relative expression of miR-483-5p and TGF- $\beta 1$ mRNA in P0, P1, and P2 chondrocytes derived from 10 different donors. The miR483-5p expression levels were significantly increased with passage number of human articular chondrocytes (Fig. 1B). By contrast, TGF- $\beta 1$ mRNA levels were marginally decreased, but no significant difference was observed (Fig. 1C). Considering the cell morphology and the expression levels of miR-483-5p and TGF- $\beta 1$, P1 chondrocytes were employed in the following experiments.

\section{miR-483-5p down-regulates TGF- $\beta 1$ expression in articular chondrocytes}

To explore the relationship between miR-483-5p and TGF- $\beta 1$ in chondrocytes, we employed qRT-PCR and western blot analyses to measure TGF- $\beta 1$ expression with miR483-5p overexpression or knock-down in P1 chondrocytes, derived from 10 different donors. MiR-483-5p mRNA expression in chondrocytes transfected with pcDNA3.1-primiR-483-5p was significantly up-regulated compared to that in cells transfected with empty pcDNA3.1 vector (pcDNA3.1-NC) (Fig. 2A). Besides, chondrocytes transfected with miR-483-5p siRNA exhibited dramatically lower miR-483-5p level than cells transfected with scramble siRNA (Fig. 2C). MiR-483-5p overexpression resulted in great reduction of TGF- $\beta 1$ expression, both in mRNA (Fig. 2A) and protein levels (Fig. 2B). By contrast, miR483-5p silencing increased TGF- $\beta 1$ expression moderately at mRNA level (Fig. 2C) but, more notably, at protein level (Fig. 2D).

miR-483-5p reduces levels of COL2A1 and aggrecan and increases levels of RUNX2 and $M M P-13$

To assess the role of miR-483-5p in chondrocytes, we evaluated the levels of key cartilage genes with miR-483-5p overexpression in human articular chondrocytes. Our results demonstrated that the expression levels of type II collagen alpha 1 (COL2A1) and aggrecan mRNAs were significantly reduced by miR-483-5p overexpression (Fig. $3 \mathrm{~A}, \mathrm{~B})$. COL2A1 and aggrecan are critical cartilage extracellular matrix proteins that are essential for normal cartilage function.

Chondrocyte hypertrophy is a crucial step in cartilage degeneration. Runt-related transcription factor 2 (RUNX2) is an important transcription factor involved in chondrocyte differentiation and promotes hypertrophy, leading to elevated levels of type II collagen-degrading matrix metalloproteinase13 (MMP13) (Wang et al. 2004), which contributes to cartilage degradation and osteoarthritis. MMP-13 is also one of the most widely used markers of hypertrophic 
A

Po

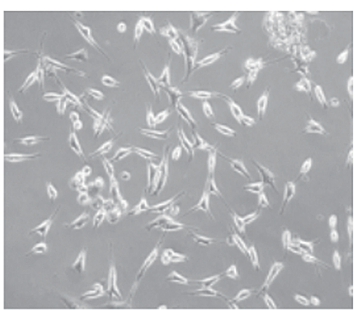

P1

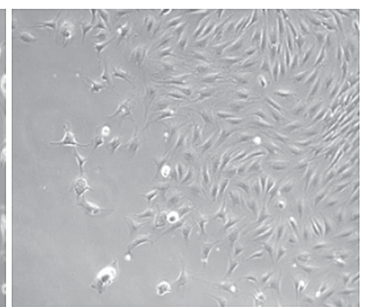

P2

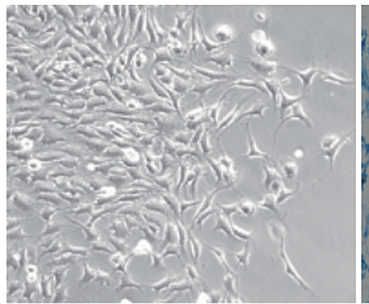

TB

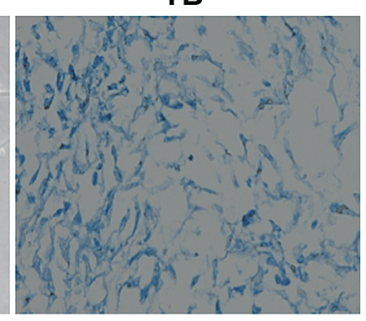

B

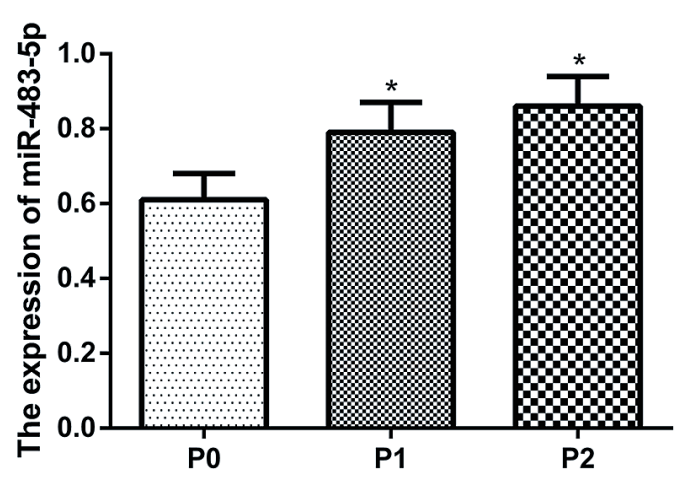

C

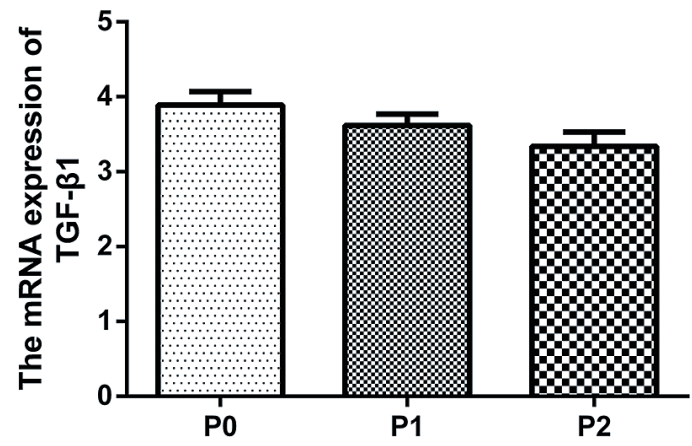

Fig. 1. Expression of miR-483-5p and TGF- $\beta 1$ in articular chondrocytes.

(A) Chondrocytes that passaged within three generations ( $\mathrm{P} 0, \mathrm{P} 1$, and $\mathrm{P} 2)$ were subcultured in vitro in this study. The first three images showed representative morphology of P0 chondrocytes cultured for $72 \mathrm{~h}, \mathrm{P} 1$, and P2 chondrocytes under inverted microscope, respectively. The last image showed the identification of representative P2 chondrocytes stained by toluidine blue (TB). (B) miR-483-5p expression and (C) mRNA expression of TGF- $\beta 1$ in P0, P1, P2 chondrocytes obtained from ten different donors $(\mathrm{n}=10)$ were detected by qRT-PCR. Values represented the average of 10 different experiments. GAPDH served as the internal control. " $P<0.05$ vs. P0 group.

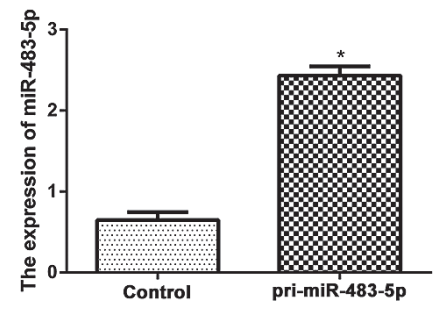

C

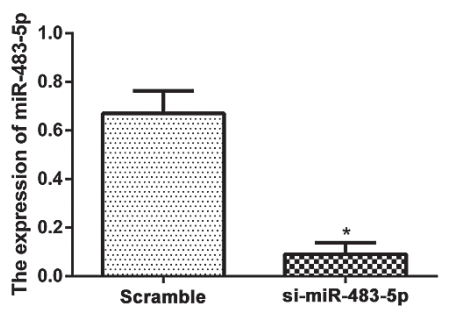

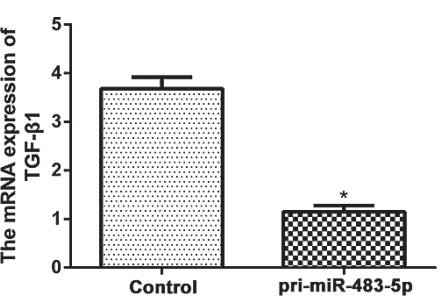

B
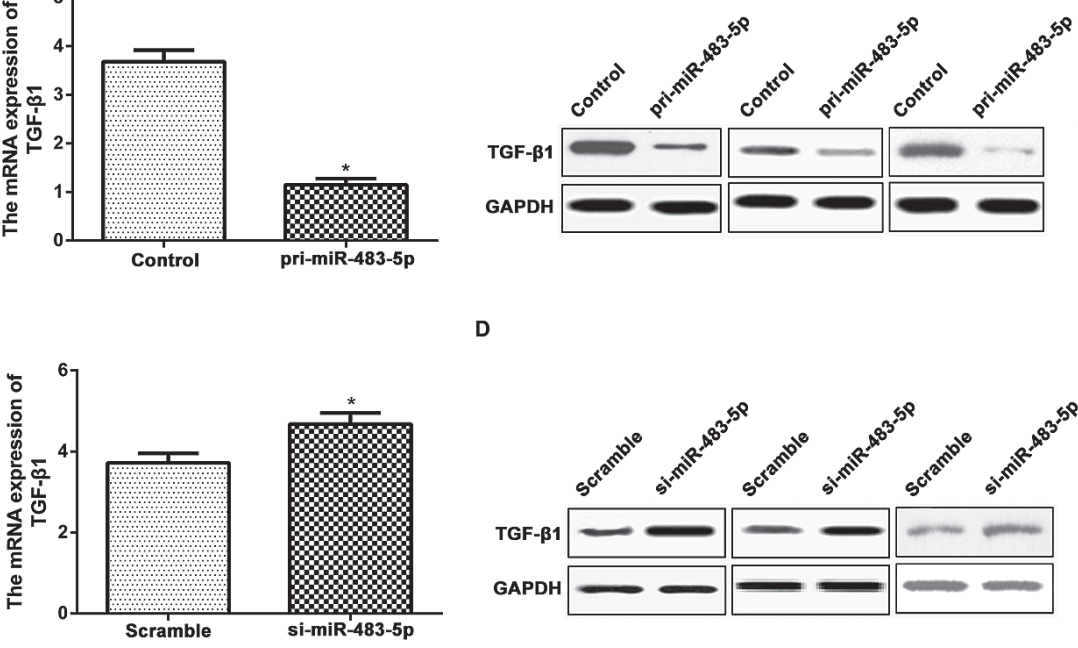

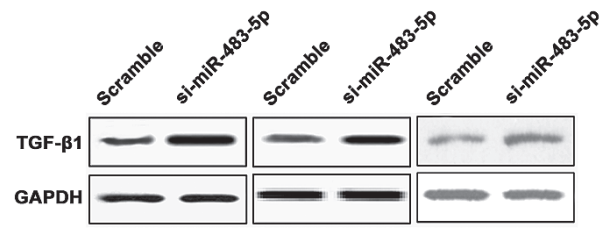

Fig. 2. miR-483-5p negatively regulates TGF- $\beta 1$ expression in human articular chondrocytes. (A) Expression of miR-483$5 \mathrm{p}$ and TGF- $\beta 1$ in P1 chondrocytes transfected with pcDNA3.1-NC (control) or pcDNA3.1-pri-miR-483-5p was assessed by qRT-PCR. Values represented the average of 10 different experiments. (B) The protein expression of TGF- $\beta 1$ in representative chondrocytes $(n=3)$ transfected with pcDNA3.1-NC or pcDNA3.1-pri-miR-483-5p was examined by Western blot. (C) Expression of miR-483-5p and TGF- $\beta 1$ in P1 cells transfected with scramble siRNA or si-miR-483-5p was assessed by qRT-PCR. Values represented the average of 10 different experiments. (D) The protein expression of TGF- $\beta 1$ in representative chondrocytes $(n=3)$ transfected with scramble siRNA or si-miR-483-5p was examined by Western blot. GAPDH served as the internal control. ${ }^{*} P<0.05$ vs. control or scramble group. 
A

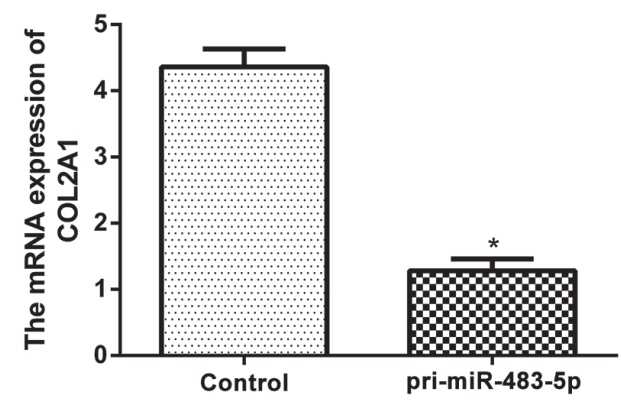

C

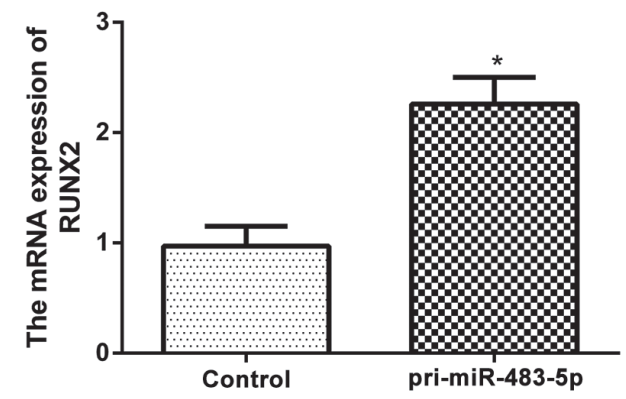

B

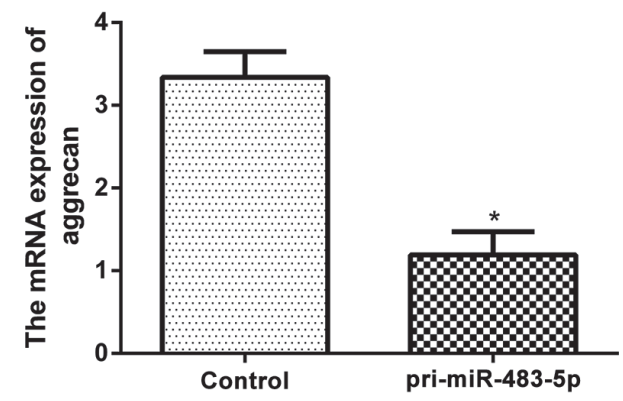

D

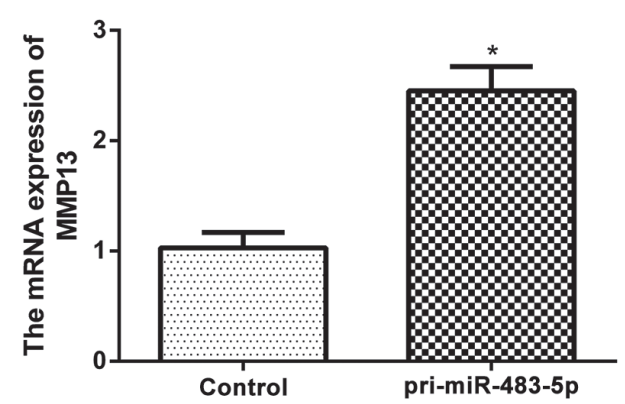

Fig. 3. miR-483-5p reduces levels of COL2A1 and aggrecan, increases levels of RUNX2 and MMP13. (A) Relative mRNA expression of COL2A1, (B) aggrecan, (C) RUNX2, and (D) MMP13 in P1 chondrocytes transfected with pcDNA3.1NC (control) or pcDNA3.1-pri-miR-483-5p were assessed by qRT-PCR. Values of qRT-PCR were averaged from ten different donors $(\mathrm{n}=10)$. GAPDH served as the internal control. ${ }^{*} P<0.05$ vs. control group.

chondrocytes (Tuckermann et al. 2000). Interestingly, in this study, these two genes were both increased by miR483-5p overexpression (Fig. 3C, D), suggesting the promotion of cartilage degeneration by overexpressing miR$483-5 \mathrm{p}$.

miR-483-5p regulates key cartilage gene expression in human articular chondrocytes through down-regulating TGF- $\beta 1$ expression

To further evaluate the underlying mechanism by which miR-483-5p regulates chondrocyte function, the expression of RUNX2 and MMP-13, as well as COL2A1 and aggrecan were evaluated with either miR-483-5p or TGF- $\beta 1$ overexpressed (Fig. 4A). The results showed that RUNX2 and MMP13 were significantly increased, COL2A1 and aggrecan were significantly decreased by overexpressing miR-483-5p, whereas TGF- $\beta 1$ overexpression dramatically reversed the levels of RUNX2, MMP13, COL2A1 and aggrecan induced by miR-483-5p overexpression (Fig. 4B, C). Above findings showed that miR-483-5p and TGF- $\beta 1$ exerted the opposite effect on key cartilage genes in human articular chondrocytes. Given the negative regulation of TGF- $\beta 1$ expression by miR-483-5p in human articular chondrocytes, we concluded that miR-483-5p elevated RUNX2 and MMP13, and reduced COL2A1 and aggrecan expression in human chondrocytes may be mediated through down-regulating TGF- $\beta 1$ expression.

\section{Discussion}

Our study provided evidence that miR-483-5p regulated cartilage degeneration via negatively regulating TGF$\beta 1$ expression. First, qRT-PCR results showed that miR$483-5$ p was significantly increased, whereas TGF- $\beta 1$ was slightly decreased and no significant difference was found in human chondrocytes from Passage 0 to Passage 2. Then we further found that miR-483-5p negatively regulated TGF- $\beta 1$ expression. In addition, miR- $483-5 p$ overexpression down-regulated critical cartilage extracellular matrix genes COL2A1 and aggrecan, and up-regulated the undesirable hypertrophy markers RUNX2 and MMP13. Given the negative regulation of TGF- $\beta 1$ expression by miR- $483-5 p$, we concluded that miR-483-5p modulated key cartilage genes in human articular chondrocytes may through downregulating TGF- $\beta 1$ expression.

Maintaining cellular morphology is vital for cartilage tissue regeneration. Articular chondrocytes will display phenotypic or morphological changes and lose their ability of cartilage tissue regeneration after the fourth passage due to rapid dedifferentiation and senescence, (Kang et al. 2007; Caron et al. 2012; Cha et al. 2013; Ashraf et al. 2016). Therefore, chondrocytes that passaged within three generations ( $\mathrm{P} 0, \mathrm{P} 1$, and $\mathrm{P} 2)$ were sub-cultured in vitro in this study.

MiR-483-5p was located within intron 2 of insulin-like growth factor 1 (IGF2) and down-regulated IGF2 expres- 

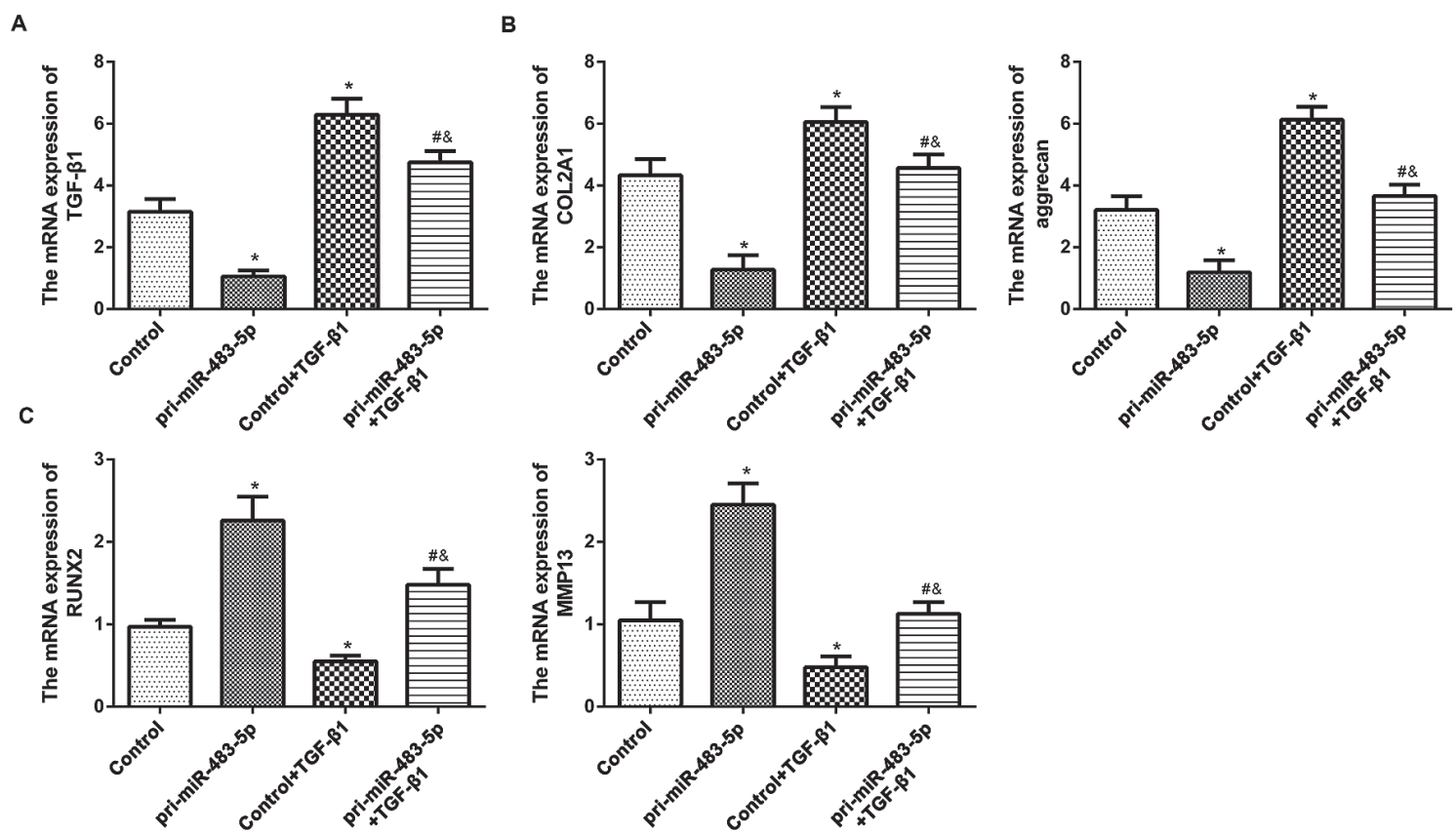

Fig. 4. miR-483-5p may regulate key cartilage genes in human articular chondrocytes through its inhibition of TGF- $\beta 1$. (A) Relative mRNA expression of TGF- $\beta 1$, (B) COL2A1, aggrecan, (C) RUNX2, and MMP13 in P1 chondrocytes transfected with pcDNA3.1-NC (control), or pcDNA3.1-pri-miR-483-5p, or co-transfected with pcDNA3.1-NC and pcDNA3.1-TGF- $\beta 1$, or co-transfected with pcDNA3.1-pri-miR-483-5p and pcDNA3.1-TGF- $\beta 1$ were assessed by qRTPCR. Values of qRT-PCR were averaged from ten different donors $(\mathrm{n}=10)$. GAPDH served as the internal control. ${ }^{*} P$ $<0.05$ vs. control group. ${ }^{\sharp} P<0.05$ vs. pri-miR-483-5p group. ${ }^{\&} P<0.05$ vs. control+TGF- $\beta 1$ group.

sion (De-Ugarte et al. 2015). MiR-483-5p was associated with various diseases including cancers, such as adrenocortical cancer (Chabre et al. 2013), colorectal cancer (Cui et al. 2016), and lung adenocarcinoma (Song et al. 2014). Interestingly, seven human miRNAs were found to show a statistically significant differential expression in human chondrocytes via evaluation of 723 miRNAs detected by miRNA microarray analysis. Amongst them, miR-483-5p was the only found to be up-regulated in osteoarthritic chondrocytes and predicted to be associated with TGF- $\beta$ signaling pathway (Díaz-Prado et al. 2012). TGF- $\beta 1$ has been reported for its beneficial effects on cartilage repair. TGF- $\beta 1$ is considered essential for cartilage integrity and is expressed at high level in normal cartilage but at reduced level in osteoarthritis cartilage (Blaney Davidson et al. 2006). TGF- $\beta 1$ stimulated the secretion of superficial zone protein (SZP), the accumulation of which at cartilage surface is important for joint homeostasis (Miyatake et al. 2016). Here we showed that the expression levels of miR$483-5 \mathrm{p}$ were increased with increasing passage number, while the expression levels of TGF- $\beta 1$ mRNA were marginally decreased. We also found that TGF- $\beta 1$ expression was negatively regulated by miR-483-5p. The identification of miR-483-5p as an upstream regulator of TGF- $\beta 1$ is critical to our understanding of cartilage homeostasis and the development of novel therapeutic approaches to cartilage repair.

Articular chondrocyte lost their differentiated phenotype and obtained a behavior with similarities to terminal differentiating chondrocytes (hypertrophy-like). Chondrocyte hypertrophy is a crucial step in cartilage degeneration and osteoarthritis pathogenesis (Chen et al. 2016). Inhibition of chondrocyte hypertrophy might be a therapeutic target to slow down cartilage degeneration and further osteoarthritis progression (van der Kraan and van den Berg 2012). RUNX2 and MMP13 (also known as collagenase 3) are the common markers of hypertrophic chondrocytes. RUNX2 is an important transcription factor involved in chondrocyte differentiation and completely controls chondrocyte hypertrophic differentiation (Stricker et al. 2002; van der Kraan and van den Berg 2012). Transgenic mice overexpressing RUNX2 in the cartilage promoted chondrocyte hypertrophic maturation and endochondral ossification, whereas inhibiting or eliminating RUNX2 activity delayed or blocked chondrocyte hypertrophic maturation (Wang et al. 2004). RUNX2 promoted hypertrophy, leading to elevated levels of MMP13 (Billinghurst et al. 1997; Wang et al. 2004), which exhibited cartilage collagenolytic activity and stimulated the degradation of type II collagen (Ishiguro and Kojima 2004; Philipot et al. 2014). MMP-13 is significantly higher at expression level in the chondrocytes of late stage osteoarthritis cartilage compared with early osteoarthritis or normal knee cartilage and contributes to osteoarthritis cartilage degradation (Bau et al. 2002; Wang et al. 2004). Transgenic mice overexpressing activated MMP-13 in their articular chondrocytes developed joint degeneration similar to human osteoarthritis (Neuhold et al. 2001). Interestingly, in this study, RUNX2 and MMP-13 were both 
increased by miR-483-5p overexpression, suggesting the promotion of cartilage degeneration by overexpressing miR-483-5p, while TGF- $\beta 1$ overexpression decreased RUNX2 and MMP-13 levels.

Aggrecan and COL2A1 are proliferative chondrocyte makers that essential to normal cartilage function. TGF- $\beta 1$ promotes osteogenesis and chondrogenesis, and enhances the synthesis of extracellular matrix protein, including aggrecan, type II collagen, and cartilage oligomeric matrix protein (Hiraki et al. 1988; Morales and Roberts 1988; Redini et al. 1988; Galera et al. 1992; Recklies et al. 1998; Wang et al. 2014; Murphy et al. 2015; Yoon et al. 2015). Overexpression of TGF- $\beta 1$ improved chondrocytes phenotype, function, and proliferation, even after several generations (Tang et al. 2017). Here, our study showed that TGF$\beta 1$ overexpression increased levels of COL2A1 and aggrecan, while miR-483-5p overexpression exerted the opposite effect on these two cartilage-related genes. Furthermore, chondrocytes simultaneously overexpressing miR-483-5p and TGF- $\beta 1$ showed no significant difference compared to the control group in the levels of RUNX2, MMP13, COL2A1 and aggrecan, suggesting that TGF- $\beta 1$ overexpression dramatically reversed the levels of RUNX2, MMP13, COL2A1 and aggrecan induced by miR-483-5p overexpression COL2A1 and aggrecan.

In summary, this study demonstrated that miR-483-5p decreased COL2A1 and aggrecan, but increased RUNX2 and MMP13, through at least partial negative regulation of TGF- $\beta 1$ expression, suggesting the possibility of inhibiting miR-483-5p to control cartilage degeneration. This may give a novel and feasible therapeutic target for cartilage repair.

\section{Conflict of Interest}

These authors declare no conflict of interest.

\section{References}

Ashraf, S., Cha, B.H., Kim, J.S., Ahn, J., Han, I., Park, H. \& Lee, S.H. (2016) Regulation of senescence associated signaling mechanisms in chondrocytes for cartilage tissue regeneration. Osteoarthritis Cartilage, 24, 196-205.

Bartel, D.P. (2004) MicroRNAs: genomics, biogenesis, mechanism, and function. Cell, 116, 281-297.

Bau, B., Gebhard, P.M., Haag, J., Knorr, T., Bartnik, E. \& Aigner, T. (2002) Relative messenger RNA expression profiling of collagenases and aggrecanases in human articular chondrocytes in vivo and in vitro. Arthritis Rheum., 46, 2648-2657.

Billinghurst, R.C., Dahlberg, L., Ionescu, M., Reiner, A., Bourne, R., Rorabeck, C., Mitchell, P., Hambor, J., Diekmann, O., Tschesche, H., Chen, J., Van Wart, H. \& Poole, A.R. (1997) Enhanced cleavage of type II collagen by collagenases in osteoarthritic articular cartilage. J. Clin. Invest., 99, 15341545.

Blaney Davidson, E.N., van der Kraan, P.M. \& van den Berg, W.B. (2007) TGF- $\beta$ and osteoarthritis. Osteoarthritis Cartilage, 15, 597-604.

Candela, M.E., Yasuhara, R., Iwamoto, M. \& Enomoto-Iwamoto, M. (2014) Resident mesenchymal progenitors of articular cartilage. Matrix Biol., 39, 44-49.

Caron, M.M., Emans, P.J., Coolsen, M.M., Voss, L., Surtel, D.A.,
Cremers, A., van Rhijn, L.W. \& Welting, T.J. (2012) Redifferentiation of dedifferentiated human articular chondrocytes: comparison of 2D and 3D cultures. Osteoarthritis Cartilage, 20, 1170-1178.

Blaney Davidson, E.N., Vitters, E.L., van der Kraan, P.M. \& van den Berg, W.B. (2006) Expression of transforming growth factor- $\beta(\mathrm{TGF} \beta)$ and the TGF $\beta$ signalling molecule SMAD-2P in spontaneous and instability-induced osteoarthritis: role in cartilage degradation, chondrogenesis and osteophyte formation. Ann. Rheum. Dis., 65, 1414-1421.

Cha, B.H., Lee, J.S., Kim, S.W., Cha, H.J. \& Lee, S.H. (2013) The modulation of the oxidative stress response in chondrocytes by Wip 1 and its effect on senescence and dedifferentiation during in vitro expansion. Biomaterials, 34, 2380-2388.

Chabre, O., Libe, R., Assie, G., Barreau, O., Bertherat, J., Bertagna, X., Feige, J.J. \& Cherradi, N. (2013) Serum miR-483-5p and miR-195 are predictive of recurrence risk in adrenocortical cancer patients. Endocr. Relat. Cancer, 20, 579-594.

Chen, W., Sheng, P., Huang, Z., Meng, F., Kang, Y., Huang, G., Zhang, Z., Liao, W. \& Zhang, Z. (2016) MicroRNA-381 regulates chondrocyte hypertrophy by inhibiting histone deacetylase 4 expression. Int. J. Mol. Sci., 17, 1377.

Cui, H., Liu, Y., Jiang, J., Liu, Y., Yang, Z., Wu, S., Cao, W., Cui, I.H. \& Yu, C. (2016) IGF2-derived miR-483 mediated oncofunction by suppressing DLC-1 and associated with colorectal cancer. Oncotarget, 7, 48456-48466.

Davies, L.C., Blain, E.J., Gilbert, S.J., Caterson, B. \& Duance, V.C. (2008) The potential of IGF-1 and TGF $\beta 1$ for promoting "adult" articular cartilage repair: an in vitro study. Tissue Eng. Part A, 14, 1251-1261.

De-Ugarte, L., Yoskovitz, G., Balcells, S., Guerri-Fernandez, R., Martinez-Diaz, S., Mellibovsky, L., Urreizti, R., Nogues, X., Grinberg, D., Garcia-Giralt, N. \& Diez-Perez, A. (2015) MiRNA profiling of whole trabecular bone: identification of osteoporosis-related changes in MiRNAs in human hip bones. BMC Med. Genomics, 8, 75.

Díaz-Prado, S., Cicione, C., Muiños-López, E., Hermida-Gómez, T., Oreiro, N., Fernández-López, C. \& Blanco, F.J. (2012) Characterization of microRNA expression profiles in normal and osteoarthritic human chondrocytes. BMC Musculoskelet. Disord., 13, 144.

Galera, P., Vivien, D., Pronost, S., Bonaventure, J., Redini, F., Loyau, G. \& Pujol, J.P. (1992) Transforming growth factor- $\beta 1$ (TGF- $\beta 1$ ) up-regulation of collagen type II in primary cultures of rabbit articular chondrocytes (RAC) involves increased mRNA levels without affecting mRNA stability and procollagen processing. J. Cell. Physiol., 153, 596-606.

Goessler, U.R., Bugert, P., Bieback, K., Deml, M., Sadick, H., Hormann, K. \& Riedel, F. (2005) In-vitro analysis of the expression of TGF $\beta$-superfamily-members during chondrogenic differentiation of mesenchymal stem cells and chondrocytes during dedifferentiation in cell culture. Cell. Mol. Biol. Lett., 10, 345-362.

Hiraki, Y., Inoue, H., Hirai, R., Kato, Y. \& Suzuki, F. (1988) Effect of transforming growth factor $\beta$ on cell proliferation and glycosaminoglycan synthesis by rabbit growth-plate chondrocytes in culture. Biochim. Biophys. Acta, 969, 91-99.

Ishiguro, N. \& Kojima, T. (2004) Role of aggrecanase and MMP in cartilage degradation. Clin. Calcium, 14, 38-44.

Kang, S.W., Yoo, S.P. \& Kim, B.S. (2007) Effect of chondrocyte passage number on histological aspects of tissue-engineered cartilage. Biomed. Mater. Eng., 17, 269-276.

Li, F., Ma, N., Zhao, R., Wu, G., Zhang, Y., Qiao, Y., Han, D., Xu, Y., Xiang, Y., Yan, B., Jin, J., Lv, G., Wang, L., Xu, C., Gao, X. \& Luo, S. (2014) Overexpression of miR-483-5p/3p cooperate to inhibit mouse liver fibrosis by suppressing the TGF- $\beta$ stimulated HSCs in transgenic mice. J. Cell. Mol. Med., 18, 966-974.

Li, X., Du, M., Liu, X., Chen, W., Wu, M., Lin, J. \& Wu, G. (2010) 
Millimeter wave treatment promotes chondrocyte proliferation by upregulating the expression of cyclin-dependent kinase 2 and cyclin A. Int. J. Mol. Med., 26, 77-84.

Liang, Z.J., Zhuang, H., Wang, G.X., Li, Z., Zhang, H.T., Yu, T.Q. \& Zhang, B.D. (2012) MiRNA-140 is a negative feedback regulator of MMP-13 in IL- $1 \beta$-stimulated human articular chondrocyte C28/I2 cells. Inflamm. Res., 61, 503-509.

Martinez-Sanchez, A., Dudek, K.A. \& Murphy, C.L. (2012) Regulation of human chondrocyte function through direct inhibition of cartilage master regulator SOX9 by microRNA-145 (miRNA-145). J. Biol. Chem., 287, 916-924.

Miyatake, K., Iwasa, K., McNary, S.M., Peng, G. \& Reddi, A.H. (2016) Modulation of superficial zone protein/lubricin/PRG4 by kartogenin and transforming growth factor- $\beta 1$ in surface zone chondrocytes in bovine articular cartilage. Cartilage, 7 , 388-397.

Morales, T.I. \& Roberts, A.B. (1988) Transforming growth factor beta regulates the metabolism of proteoglycans in bovine cartilage organ cultures. J. Biol. Chem., 263, 12828-12831.

Murphy, M.K., Huey, D.J., Hu, J.C. \& Athanasiou, K.A. (2015) TGF- $\beta 1$, GDF-5, and BMP-2 stimulation induces chondrogenesis in expanded human articular chondrocytes and marrowderived stromal cells. Stem Cells, 33, 762-773.

Neuhold, L.A., Killar, L., Zhao, W., Sung, M.L., Warner, L., Kulik, J., Turner, J., Wu, W., Billinghurst, C., Meijers, T., Poole, A.R., Babij, P. \& DeGennaro, L.J. (2001) Postnatal expression in hyaline cartilage of constitutively active human collagenase-3 (MMP-13) induces osteoarthritis in mice. J. Clin. Invest., 107, 35-44.

Patterson, E.E., Holloway, A.K., Weng, J., Fojo, T. \& Kebebew, E. (2011) MicroRNA profiling of adrenocortical tumors reveals miR-483 as a marker of malignancy. Cancer, 117, 1630-1639.

Philipot, D., Guerit, D., Platano, D., Chuchana, P., Olivotto, E., Espinoza, F., Dorandeu, A., Pers, Y.M., Piette, J., Borzi, R.M., Jorgensen, C., Noel, D. \& Brondello, J.M. (2014) p16 ${ }^{\mathrm{INK} 4 \mathrm{a}}$ and its regulator miR-24 link senescence and chondrocyte terminal differentiation-associated matrix remodeling in osteoarthritis. Arthritis Res. Ther., 16, R58.

Qureshi, H.Y., Ahmad, R., Sylvester, J. \& Zafarullah, M. (2007) Requirement of phosphatidylinositol 3-kinase/Akt signaling pathway for regulation of tissue inhibitor of metalloproteinases-3 gene expression by TGF- $\beta$ in human chondrocytes. Cell. Signal., 19, 1643-1651.

Recklies, A.D., Baillargeon, L. \& White, C. (1998) Regulation of cartilage oligomeric matrix protein synthesis in human synovial cells and articular chondrocytes. Arthritis Rheum., 41, 997-1006.

Redini, F., Galera, P., Mauviel, A., Loyau, G. \& Pujol, J.P. (1988) Transforming growth factor beta stimulates collagen and glycosaminoglycan biosynthesis in cultured rabbit articular chondrocytes. FEBS Lett., 234, 172-176.

Song, Q., Xu, Y., Yang, C., Chen, Z., Jia, C., Chen, J., Zhang, Y., Lai, P., Fan, X., Zhou, X., Lin, J., Li, M., Ma, W., Luo, S. \& Bai, X. (2014) miR-483-5p promotes invasion and metastasis of lung adenocarcinoma by targeting RhoGDI1 and ALCAM. Cancer Res., 74, 3031-3042.

Stricker, S., Fundele, R., Vortkamp, A. \& Mundlos, S. (2002) Role of Runx genes in chondrocyte differentiation. Dev. Biol., 245, 95-108.

Tang, Y., Xiao, J., Wang, Y., Li, M. \& Shi, Z. (2017) Effect of adenovirus-mediated TGF- $\beta 1$ gene transfer on the function of rabbit articular chondrocytes. J. Orthop. Sci., 22, 149-155.

Tuckermann, J.P., Pittois, K., Partridge, N.C., Merregaert, J. \& Angel, P. (2000) Collagenase-3 (MMP-13) and Integral Membrane Protein 2a (Itm 2a) are Marker Genes of Chondrogenic/Osteoblastic Cells in Bone Formation: Sequential Temporal, and Spatial Expression of Itm2a, Alkaline Phosphatase, MMP-13, and Osteocalcin in the Mouse. J. Bone Miner. Res., 15, 1257-1265.

van der Kraan, P.M. \& van den Berg, W.B. (2012) Chondrocyte hypertrophy and osteoarthritis: role in initiation and progression of cartilage degeneration? Osteoarthritis Cartilage, 20, 223-232.

Wang, W., Rigueur, D. \& Lyons, K.M. (2014) TGF $\beta$ signaling in cartilage development and maintenance. Birth Defects Res. $C$ Embryo Today, 102, 37-51.

Wang, X., Manner, P.A., Horner, A., Shum, L., Tuan, R.S. \& Nuckolls, G.H. (2004) Regulation of MMP-13 expression by RUNX2 and FGF2 in osteoarthritic cartilage. Osteoarthritis Cartilage, 12, 963-973.

Worster, A.A., Nixon, A.J., Brower-Toland, B.D. \& Williams, J. (2000) Effect of transforming growth factor betal on chondrogenic differentiation of cultured equine mesenchymal stem cells. Am. J. Vet. Res., 61, 1003-1010.

Yang, M., Zhang, L. \& Gibson, G.J. (2015) Chondrocyte miRNAs 221 and 483-5p respond to loss of matrix interaction by modulating proliferation and matrix synthesis. Connect. Tissue Res., 56, 236-243.

Yoon, H.J., Kim, S.B., Somaiya, D., Noh, M.J., Choi, K.B., Lim, C.L., Lee, H.Y., Lee, Y.J., Yi, Y. \& Lee, K.H. (2015) Type II collagen and glycosaminoglycan expression induction in primary human chondrocyte by TGF- $\beta 1$. BMC Musculoskelet. Disord., 16, 141.

Yu, X., Zhang, X., Bi, T., Ding, Y., Zhao, J., Wang, C., Jia, T., Han, D., Guo, G., Wang, B., Jiang, J. \& Cui, S. (2013) MiRNA expression signature for potentially predicting the prognosis of ovarian serous carcinoma. Tumour Biol., 34, 3501-3508.

Zhu, Y., Tao, H., Jin, C., Liu, Y., Lu, X., Hu, X. \& Wang, X. (2015) Transforming growth factor- $\beta 1$ induces type II collagen and aggrecan expression via activation of extracellular signalregulated kinase 1/2 and Smad2/3 signaling pathways. Mol. Med. Rep., 12, 5573-5579.

Zuntini, M., Salvatore, M., Pedrini, E., Parra, A., Sgariglia, F., Magrelli, A., Taruscio, D. \& Sangiorgi, L. (2010) MicroRNA profiling of multiple osteochondromas: identification of disease-specific and normal cartilage signatures. Clin. Genet., 78, 507-516. 